\title{
Elucidação Estrutural Através da Cristalografia: Teoria Básica
}

\author{
Jean Marcos F. Custodio, Luiz F. N. Naves \& Wesley F. Vaz
}

\begin{abstract}
Quando é necessário identificar e/ou analisar a matéria e sua estrutura tridimensional, a cristalografia surge como uma poderosa solução metodológica. Ela se baseia no experimento da difração dos raios-x por cristais e é amplamente utilizada em várias áreas das ciências. Baseados na aplicação da cristalografia como ferramenta que leva ao conhecimento estrutural molecular, nós propomos aqui um maior detalhamento da teoria básica envolvida em uma análise cristalográfica. Foram abordados os três pilares fundamentais da cristalografia (simetria, difração de raios-x e densidade eletrônica), partindo da degenerescência dos sistemas cristalinos, passando pelo experimento de difração e a lei de Bragg, transformada de Fourier e, finalmente, o problema da fase.
\end{abstract}

Palavras-Chave: cristalografia; simetria; difração de raios-x.

When it is necessary to identify and/or analyze the matter and its three-dimensional structure, crystallography emerges as a powerful methodological solution. It is based on the experiment of x-ray diffraction by crystalline samples and is widely used in several fields of sciences. Based on the application of crystallography as a tool that leads to molecular structural knowledge, we propose here a greater detail of the basic theory involved in a crystallographic analysis. The three fundamental pillars of crystallography (symmetry, $\mathrm{x}$ - ray diffraction and electron density) were studied, starting from the degeneracy of the crystalline systems, through the diffraction experiment and the Bragg law, Fourier transform and, finally, the phase problem.

Keywords: crystallography; symmetry; $x$ - ray diffraction. 


\section{Introdução}

A cristalografia estuda a matéria em seu estado sólido ou condensado, estado no qual o movimento das moléculas é restrito devido às fortes interações intermoleculares ali presentes, resultando em uma estrutura rígida. As propriedades dos sólidos dependem do tipo de força existente entre os átomos/moléculas ${ }^{1}$. Um cristal pode ser definido como um sólido composto de átomos, dispostos segundo um padrão periódico em três dimensões. Como tal, cristais diferem de uma maneira fundamental de gases e líquidos, porque os arranjos atômicos no segundo grupo não possuem o requisito essencial de periodicidade. Nem todos os sólidos são cristalinos, no entanto, alguns são amorfos, como o vidro, e não têm qualquer arranjo interior regular de átomos ${ }^{2}$.

Uma característica importante repetida no interior de cristais reais é a distribuição da densidade eletrônica, devido a isso, os raios- $\mathrm{X}$ incidentes podem interagir com um elétron do cristal e gerar padrões de interferência de raios-X. A simetria do padrão de interferência contém informações sobre a ordem interna do cristal (a "ordem interna tridimensional" por definição), enquanto que as intensidades dos feixes difratados contêm informações estruturais sobre o objeto a ser repetido (o "sólido homogêneo" por definição). Por conseguinte, a fim de compreender a análise da estrutura de um cristal, um entendimento detalhado de simetria é importante. Dessa maneira, as próximas seções discorrerão com mais acerca da simetria em compostos cristalinos e, posteriormente, sobre a difração de raios-X em tais compostos ${ }^{3}$.

\section{CELA UNITÁRIA E REDES DE BRAVAIS}

Em um cristal, a repetição de um objeto em uma orientação idêntica (ou seja, sem qualquer rotação) em intervalos regulares é caracterizada por três vetores não coplanares, denominados eixos cristalográficos, representados por a, b e c. Esses vetores são escolhidos de modo que, tomados em sequência, formam um sistema axial que segue a regra da mão direita. Posições atômicas, ao longo de cada uma dessas direções, são geralmente medidas em termos de coordenadas fracionárias $\mathrm{x}, \mathrm{y}$ e $\mathrm{z}$ variando de 0 a 1 . Os ângulos formados entre os vetores axiais são designados por $\gamma$ (entre a e b), $\beta$ (entre a e c) e $\alpha$ (entre b e c). A unidade do padrão repetida regularmente por translação em uma substância cristalina é chamada de "cela unitária" daquele cristal, que pode também ser definida como a menor porção do cristal que ainda conserva as propriedades originais desse. As seis dimensões da cela unitária, a, b, c, $\alpha, \beta$ e $\gamma$ determinam sua forma e o seu tamanho. Por convenção, a cela unitária é escolhida de modo a exibir a maior simetria do cristal e terá ângulos interaxiais não muito distantes de $90^{\circ}$. Uma representação de cela unitária é dada na Figura 1(a)

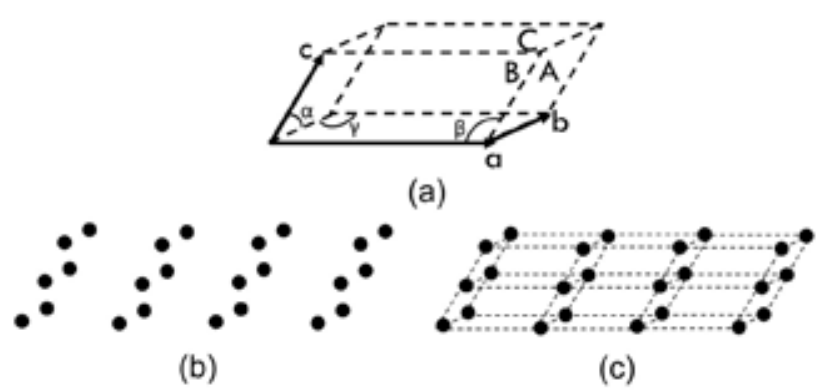

Figura 1. Representação de uma cela unitária em (a) mostrando os eixos cristalográficos a, b e c, os ângulos interaxiais $\alpha, \beta$ e $\gamma$ e as faces A, B e C. Em (b) é mostrada uma base formada por pontos e, por fim, em (c) é mostrada a rede cristalina da base (b).

Ao descrever sistemas cristalinos, muitas vezes, é conveniente ignorar os átomos reais que compõem o cristal e o seu arranjo periódico no espaço e substituí-los por um conjunto de pontos imaginários que possuem uma relação fixa com os átomos do cristal no espaço, podendo ser considerados como um tipo de "esqueleto" sobre o qual o cristal real é constituído. Dessa maneira, a base de um cristal em particular [Figura 1(b)] é o conjunto de pontos infinitos que podem ser gerados a partir de um ponto inicial pela repetição infinita dos vetores da cela unitária do cristal. Frequentemente, há erros conceituais como confundir o termo "base" com "estrutura" ou afirmar que a base apenas 
indica os vértices da cela unitária. $\mathrm{O}$ conjunto de pontos que caracteriza a base de um determinado cristal pode ser estabelecido sobre a estrutura desse cristal de modo que cada ponto tenha propriedades idênticas em cada cela unitária, independentemente, de onde aquele ponto possa estar. Os átomos do cristal podem estar nos pontos, mas os pontos podem muito bem estar entre os átomos. A ideia principal aqui tratada é que cada ponto na cela unitária terá o mesmo ambiente atômico em cada cela unitária. A rede cristalina é a combinação das celas unitárias e a base, como representado na Figura 1(c).

Ao dividir o espaço em três conjuntos de planos, podem ser obtidas celas unitárias das mais variadas formas, dependendo de como tais planos são arranjados. Por exemplo, se esses três planos forem igualmente espaçados e mutuamente perpendiculares, a cela unitária é cúbica. Nesse caso, os vetores $a, b$ e c são iguais, bem como os ângulos formados entre eles, ou seja, $a=b=c$ e $\alpha=\beta=\gamma^{2}$. Os sete sistemas cristalinos são obtidos pela comutação dos valores angulares e axiais, sendo listados na Tabela 1

Tabela 1. Configurações espaciais dos sete sistemas cristalinos

\begin{tabular}{|c|c|c|}
\hline Sistema cristalino & Valores axiais & Valores angulares \\
\hline Cúbico & $\mathrm{a}=\mathrm{b}=\mathrm{c}$ & $\alpha=\beta=\gamma=90^{\circ}$ \\
\hline Tetragonal & $\mathrm{a}=\mathrm{b} \neq \mathrm{c}$ & $\alpha=\beta=\gamma=90^{\circ}$ \\
\hline Ortorrômbico & $\mathrm{a} \neq \mathrm{b} \neq \mathrm{c}$ & $\alpha=\beta=\gamma=90^{\circ}$ \\
\hline $\begin{array}{c}\text { Trigonal (ou } \\
\text { romboédrico) }\end{array}$ & $\mathrm{a}=\mathrm{b}=\mathrm{c}$ & $\alpha=\beta=\gamma \neq 90^{\circ}$ \\
\hline Hexagonal & $\mathrm{a}=\mathrm{b} \neq \mathrm{c}$ & $\alpha=\beta=90^{\circ}, \gamma=120^{\circ}$ \\
\hline Monoclínico & $\mathrm{a} \neq \mathrm{b} \neq \mathrm{c}$ & $\alpha=\gamma=90^{\circ} \neq \beta$ \\
\hline Triclínico & $\mathrm{a} \neq \mathrm{b} \neq \mathrm{c}$ & $\alpha \neq \beta \neq \gamma \neq 90^{\circ}$ \\
\hline
\end{tabular}

Para a definição de cela unitária e parâmetros de rede da seção anterior, foram usados exemplos de cela unitária primitiva, isso é, apenas um ponto por cela. Cada uma dessas celas primitivas definem um tipo de base. Porém, há ainda outros tipos de base que são baseadas em celas não primitivas, independentes em relação às primitivas.

Outro tipo de cela unitária é percebido se, por exemplo, em uma cela cúbica houver pontos localizados no centro da cela, além dos vértices. Nesse caso, os novos arranjos de pontos na base alteram o tipo da cela unitária. Similarmente, um novo tipo de base é gerado em uma cela unitária cúbica na qual há pontos situados nos centros de cada face.

As quatorze redes de Bravais são representadas na Figura 2, sendo os símbolos P, F, I, etc., com os significados como seguem. A primeira distinção a ser feita é entre celas primitivas (representadas por $\mathrm{P}$ ou $\mathrm{R}$ ) e celas não-primitivas (demais símbolos): celas primitivas têm somente um ponto a cada cela unitária, enquanto que as não-primitivas possuem dois ou mais pontos por cela unitária. Um ponto no interior de uma cela pertence àquela cela, enquanto que um ponto em uma determinada face é compartilhado por duas celas e, por fim, um ponto no vértice de uma cela é compartilhado por oito celas. Dessa maneira, o número de pontos por cela é definido por

onde $\mathrm{Ni}=$ número de pontos no interior, $\mathrm{Nf}=$ número de

$$
N=N_{i}+\frac{N_{f}}{2}+\frac{N_{c}}{8}
$$

pontos nas faces e $\mathrm{Nc}=$ número de pontos nos vértices da cela unitária. Pela análise da Equação 1, torna-se claro que qualquer cela com pontos apenas nos vértices é, portanto, primitiva, enquanto uma que contém pontos adicionais no interior ou nas faces é não-primitiva. $\mathrm{O}$ símbolo $\mathrm{F}$ e I significam celas unitárias centrado nas faces e de corpo centrado, respectivamente, enquanto que os símbolos $\mathrm{A}, \mathrm{B}$ e $\mathrm{C}$ significam centrados nas faces $\mathrm{A}, \mathrm{B}$ ou $\mathrm{C}$, respectivamente. $\mathrm{O}$ símbolo $\mathrm{R}$ é usado especialmente para o sistema romboédrico (ou triclínico) ${ }^{2}$

A ausência dos demais tipos de cela unitária na Figura 2 

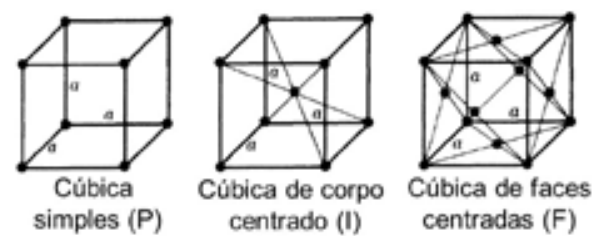

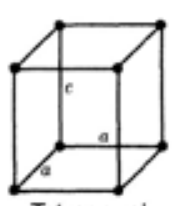

Tetragonal simples (P)

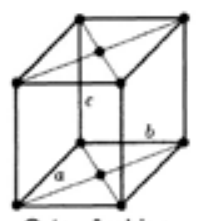

Ortorrômbica centrada em C (C)

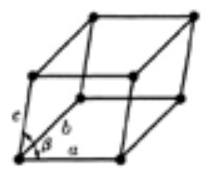

Monoclínica simples (P)

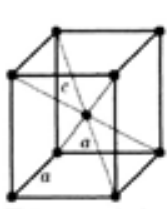

Tetragonal de corpo centrado (I)

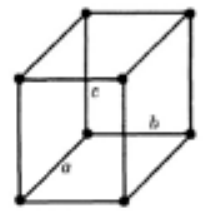

Ortorrômbica simples (P)

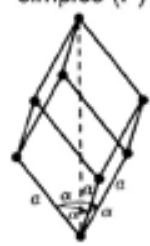

\section{Trigonal} romboédrica ( $R$ )

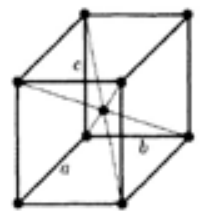

Ortorrômbica de corpo centrado (I)

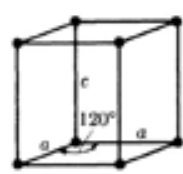

Hexagonal(P) de faces centradas (F)
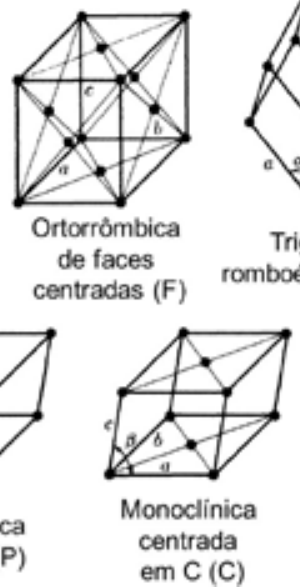

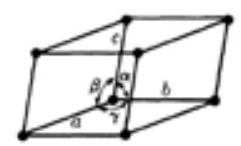

Triclínica simples (P)
Figura 2. Representação das 14 redes de Bravais. Adaptado de Cullity(2014)

(por exemplo, monoclínica de faces ou corpo centrado) por ser explicado por meio da redução de simetria celular. Por exemplo, uma cela monoclínica centrada nas faces $(\mathrm{F})$ pode ser reduzida a uma cela monoclínica centrada em $\mathrm{C}^{5}$. Por não ser o foco do escopo desse artigo, tais transformações não serão aqui abordadas.

\section{SIMETRIA PONTUAL E ESPACIAL}

A seção anterior descreveu a natureza cristalina em sua esfera externa, isto é, as características do padrão de periodicidade e escolha da cela unitária - a menor porção que se repete por translação em um composto cristalino.
Porém, elevando tal discussão a um nível interno obtêmse o padrão estrutural contido em uma única cela unitária. Nessa altura, faz-se necessário o entendimento de termos básicos na descrição da simetria pontual.

A unidade assimétrica é a menor parte de uma estrutura cristalina, a partir da qual a estrutura completa pode ser derivada através da utilização de operações de simetria nos grupos espaciais (incluindo translações). A unidade assimétrica pode ser constituída por apenas uma molécula ou íon, parte de uma molécula, ou de várias moléculas que não estão relacionadas por simetria cristalográfica. A unidade molecular é a unidade formadora da unidade assimétrica. Uma operação de simetria é a conversão de uma unidade em outra aparentemente idêntica à primeira, podendo ser em relação a um ponto, um eixo ou um plano de simetria. Assim, um objeto é simétrico quando uma operação de simetria aplicada nele produz um segundo objeto indistinguível do original. Os meios usados na operação de simetria, isso é, o ponto, eixo ou plano, são chamados de elementos de simetria ${ }^{6}$.

A simetria pontual é regida pela congruência dos objetos. Dois objetos são considerados congruentes quando pontos situados em ambos os objetos são correspondentes e distam igualmente um do outro. Consequentemente, os ângulos correspondentes serão iguais em valores absolutos. A congruência pode ser do tipo direta ou oposta em relação ao produto final. A congruência direta não inverte as propriedades do objeto ou, aplicado à química, não inverte a quiralidade da molécula. Dessa maneira, na congruência direta, o objeto age como um corpo rígido. As principais operações de simetria de congruência direta presente nos grupos pontuais são a translação e rotação. Se a congruência é oposta, então ambos os objetos envolvidos na operação de simetria são ditos enantiomorfos, uma vez que a quiralidade molecular é invertida após tal operação. As principais operações de simetria de congruência oposta presente nos grupos pontuais são a inversão, a reflexão e a rotoinversão (o produto de uma rotação seguida de uma inversão) $)^{5}$.

A Figura 3(a) mostra um paralelogramo. Uma vez que o paralelogramo possui dois pares de lados congruentes, 
esse permanece inalterado após rotações de $180^{\circ}$ ou $360^{\circ}$. $\mathrm{O}$ ângulo mínimo de rotação para que a forma seja idêntica à original é igual a $180^{\circ}$, diferente da Figura 3(c), na qual o ângulo mínimo de rotação é igual a $90^{\circ}$. Eixos de simetria rotacional são representados por $360 / \Theta=n$, em que $\Theta$ é o ângulo mínimo de rotação e $\mathrm{n}$ é conhecido como "ordem do eixo de rotação". Por definição, um eixo de rotação de ordem n é uma linha na qual o corpo é transformado em si mesmo $\mathrm{n}$ vezes durante uma rotação de $360^{\circ}$.

Os eixos de simetria rotacional de ordem 2, 3, 4 e 6 e

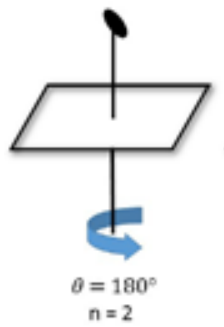

(a)

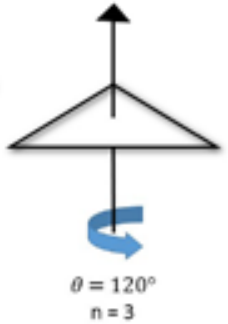

(b)

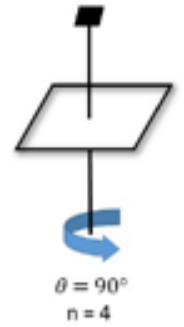

(c)

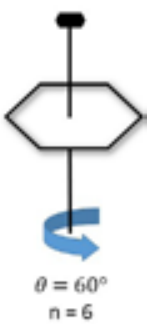

(d)
Figura 2-3: Representação dos eixos de rotação próprios de ordem 2, com $\Theta=180^{\circ}$ (a), ordem 3,

os valores angulares são mostrados na Figura 3. A partir dos eixos representados, torna-se claro que qualquer corpo regular ou não apresenta intrinsecamente um eixo de rotação de ordem 1. Eixos de rotação de ordem 5 ou superior a 6 não são possíveis, uma vez que tais formas não são capazes de perfazer completamente um plano da cela unitária. A inexistência desses eixos de rotação é definida pelo teorema da restrição cristalográfica 7 .

Outro tipo de operação de simetria é a reflexão através de um plano com espelho. Essa operação converte um objeto em sua imagem especular. A simetria de reflexão ocorre quando um plano pode ser construído tal que um objeto ou molécula de um lado do plano se relaciona ao outro como se tivesse sido resultado de uma reflexão por um espelho entre eles. Um espelho no plano paralelo ao plano yz e passando através da origem converterá um objeto em $\mathrm{x}, \mathrm{y}, \mathrm{z}$ em um objeto de coordenadas $-\mathrm{x}, \mathrm{y}, \mathrm{z}$. Um plano especular é representado pela letra $\mathrm{m}$ e ocorre naturalmente em moléculas orgânicas. Somado a isso, há também a operação de simetria que envolve a inversão, uma transformação "de dentro para fora". Um corpo tem um centro de inversão se os pontos correspondentes do corpo são localizados a distâncias iguais a partir do centro de uma linha traçada entre tais pontos. Um corpo que tem um centro de inversão terá sua configuração invertida se cada ponto no corpo é invertido, ou "refletido", no centro de inversão ${ }^{6}$. A Figura 4 mostra o centro de inversão (ou centro de simetria) de um análogo de neolignana.

A partir da combinação dos eixos de rotação próprios,

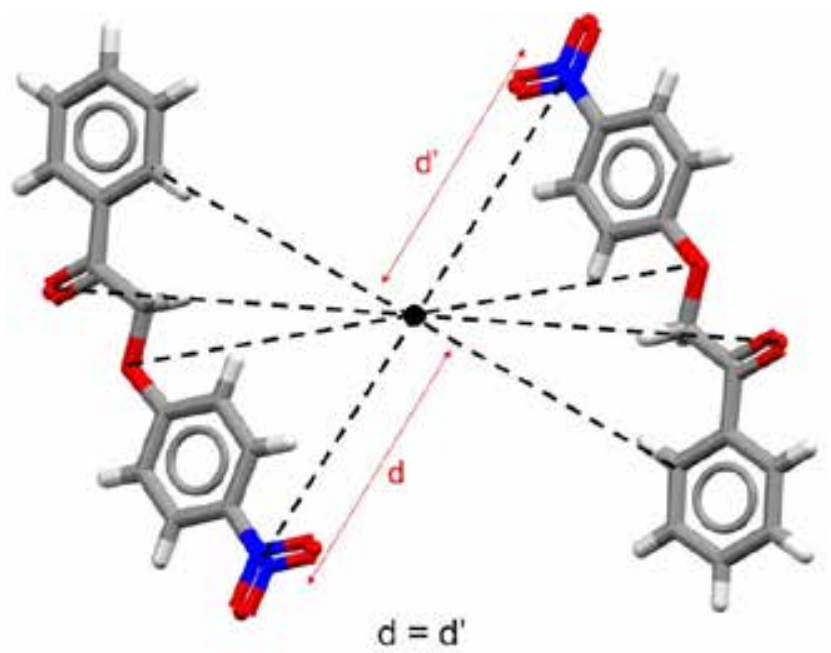

Figura 4. Representação do centro de inversão de um análogo de neolignana. d e d' são as distâncias de um ponto até o centro de simetria molecular. Note a igualdade entre d e d'.

impróprios (isto é, espelho e rotoinversões) e inversões são derivados os trinta e dois grupos pontuais (Tabela 2). Nesse ponto, é importante ressaltar que duas operações de simetria sempre irão gerar uma terceira operação de simetria. Por exemplo, se um mesmo objeto apresenta dois eixos de rotação próprio, um terceiro eixo de rotação impróprio será gerado. Entretanto, se um eixo de rotação próprio e um de 
rotação imprópria se interceptam, automaticamente, será gerado um eixo de rotação impróprio. Finalmente, um eixo de rotação próprio será gerado a partir da interceptação de dois eixos de rotação impróprios ${ }^{1}$.

Nem todas as reflexões de Bragg, I(hkl), são únicas em

Tabela 2. Os 32 grupos pontuais (com ênfase nos 11 grupos de Laue)

\begin{tabular}{ll}
\hline Sistema cristalino & Grupo pontual \\
\hline Triclinico & $1 ; \overline{1}^{*}$ \\
Monoclinico & $2 ; m ; 2 / m^{*}$ \\
Ortorrômbico & $222 ; m m 2 ; m m m^{*}$ \\
Tetragonal & $4 ; \overline{4} ; 4 / m^{*} ; 422 ; 4 m m ; \overline{4} 2 m ; 4 / \mathrm{mmm}^{*}$ \\
Trigonal/Romboédrico & $3 ; \overline{3}^{*} ; 32 ; 3 m ; \overline{3} m^{*}$ \\
Hexagonal & $6 ; \overline{6} ; 6 / m^{*} ; 622 ; 6 \mathrm{~mm} ; \overline{6} m \overline{2} ; 6 / \mathrm{mmm}^{*}$ \\
Cúbico & $23 ; m \overline{3}^{*} ; 432 ; \overline{4} 3 m ; m 3 m^{*}$
\end{tabular}

intensidade. Georges Friedel percebeu que a distribuição de intensidade em um padrão de difração é centrossimétrica, ou chamada Lei de Friedel. Como resultado, haverá reflexões de

$$
I(h k l)=I(\overline{h k l})
$$

Bragg com diferentes (mas relacionados) índices que terão intensidades idênticas. A única exceção para a Lei de Friedel é encontrada se os átomos espalharem a radiação de forma anômala.

Qualquer simetria no empacotamento de objetos é relacionada (de maneira recíproca) a simetria do seu padrão de difração, e esse último pode ser usado para determinar a simetria cristalina, a fim de obtenção do grupo espacial. A combinação dessa última informação com a lei de Friedel é de extrema importância, pois impõe um padrão de difração centrossimétrico à toda estrutura analisada, independente do cristal possuir ou não um centro de simetria. Consequentemente, o padrão de difração sempre pertencerá a um grupo pontual centrossimétrico, marcados com um asterisco na Tabela 2 e são conhecidos como grupos de Laue ${ }^{6}$.

Perfazendo todos os aspectos de simetria até agora vistos, tem-se que: (a) a métrica da cela unitária produz sete distintos sistemas cristalinos; (b) a topografia de vizinhança da cela unitária permite a existência de outros retículos cristalinos (senão o primitivo), o que causa a degenerescência dos sete sistemas cristalinos em quatorze sistemas cristalinos, denominados Redes de Bravais; e (c) a combinação das operações de simetria próprias (rotação e translação) e impróprias (reflexo, inversão e rotoinversão) resultam em um total de trinta e dois grupos pontuais, sendo os centrossimétricos denominados de grupos de Laue. Consequentemente, combinando as quatorze redes de Bravais com os trinta e dois grupos pontuais, há a ocorrência de setenta e três grupos espaciais simórficos.

Como última esfera da simetria cristalina, os grupos espaciais devem conter todas as possibilidades para o arranjo tridimensional. Para isso, são consideradas outras duas operações de simetria (produto da combinação de rotação, translação e reflexão): (a) o produto de uma rotação seguida de uma translação (eixo parafuso); e (b) o produto de uma reflexão seguida de translação (plano com deslizamento).

Um eixo parafuso, $\mathrm{nr}$, envolve uma rotação de $360^{\circ} / \mathrm{n}$ sobre um eixo combinado com uma translação paralela ao mesmo eixo por $\mathrm{r} / \mathrm{n}$ da cela unitária naquela direção. Um eixo parafuso 2 que passa pela origem da cela unitária e é paralelo a $b$ converte um objeto em $\mathrm{x}, \mathrm{y}, \mathrm{z}$ em um em $-\mathrm{x}, 1 / 2+\mathrm{y},-\mathrm{z}$. A identidade enantiomórfica do objeto é mantida após essa operação de simetria. Um plano com deslizamento envolve uma reflexão em relação à um plano combinado com uma translação. Para um plano de deslizamento, denominado a, b ou c, o componente translacional é $\mathrm{a} / \mathrm{b}, \mathrm{a} / \mathrm{b}$ ou $\mathrm{c} / 2 \mathrm{ou}$, respectivamente. Para um plano com deslizamento c, se o plano do espelho é perpendicular a b e a translação é paralela a c, um objeto em $\mathrm{x}, \mathrm{y}, \mathrm{z}$ é convertido no seu enantiomorfo situado em $-\mathrm{x},-\mathrm{y}$, $1 / 2+z$. Outros tipos de planos com deslizamentos incluem um deslizamento diagonal, denominado n, com translações 
$\mathrm{a} / 2+\mathrm{b} / 2$, ou $\mathrm{a} / 2+\mathrm{c} / 2$, ou $\mathrm{b} / 2+\mathrm{c} / 2$, e um deslizamento diagonal, denominado $\mathrm{d}$, com translações $\mathrm{a} / 4+\mathrm{b} / 4$, ou a/4 $+\mathrm{c} / 4$, ou b/4 $+\mathrm{c} / 4^{6}$.

A Figura 5 mostra dois eixos parafuso de ordem $21 \mathrm{com}$ componente translacional de magnitude $1 / 2$ e direção $t$ (a) e dois planos de deslizamento c, ou seja, com translação na direção do eixo cristalográfico c (b) referentes a um análogo de neolignana.

Após a aplicação desses produtos de operações de

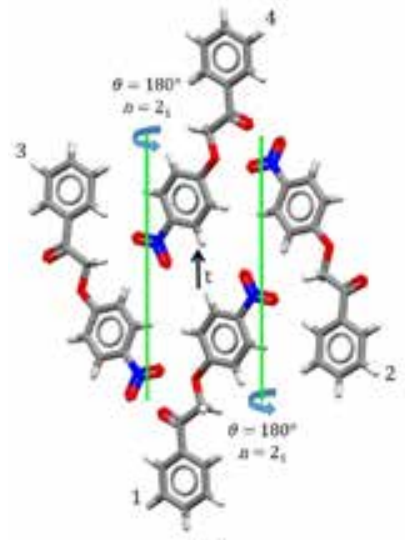

(a)

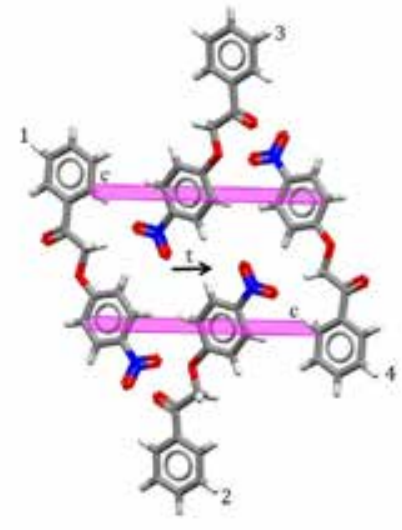

(b)
Figura 5. Representação das operações de simetria eixo helicoidal 21 (eixo parafuso) (a) e plano com deslizamento (b). Em ambas as imagens, t representa a direção da translação. Em (b), o deslizamento t é feito na direção do eixo cristalográfico c.

simetria, os setenta e três grupos espaciais iniciais se tornam, no total, duzentos e trinta grupos. Cada um desses grupos pode ser definido como o conjunto de transformações que levam à periodicidade tridimensional do arranjo cristalino. Consequentemente, um grupo espacial é o grupo de operações que convertem uma molécula ou unidade assimétrica em um padrão espacial. Os grupos espaciais podem ser identificados, embora por vezes com ambiguidade, a partir das ausências sistemáticas no padrão de difração. $\mathrm{O}$ volume $\mathrm{A}$ da Tabela Internacional de Cristalografia (International Tables for Crystallography - Vol A) ${ }^{8}$ sumariza todos os grupos espaciais aqui mencionados.

\section{Difração de Raios-X}

Há muito a análise estrutural é realizada partindo-se da interação da energia com a matéria. De uma forma sucinta e simplista, a energia contida em uma onda eletromagnética interage com a matéria e essa responde de acordo com a quantidade de energia recebida, produzindopadrões para análise estrutural. Por exemplo, na espectroscopia de infravermelho, a interação da luz com a matéria causa a absorção de energia o que, devido à energia contida na onda eletromagnética, resulta em uma deformação axial, produzindo valores específicos para determinados fragmentos moleculares ${ }^{9}$. Uma onda eletromagnética é um pulso energético que se propaga no vácuo transportando energia. Tais ondas são associadas a partículas materiais com energia dada pela Equação 3 em que h é a constante de Planck, c é a velocidade da luz,

$$
E=h v=\frac{h c}{\lambda}
$$

que corresponde à velocidade de propagação de qualquer onda eletromagnética no vácuo e $\lambda$ é o comprimento de onda ${ }^{10}$. A fim de compreender não apenas a composição química, como também a distribuição tridimensional da matéria, faz-se necessária a abordagem de uma interação tal que seja compatível com o nível microscópico da matéria. Nesse sentido, os raios-X são de extrema importância na cristalografia, uma vez que seu comprimento de onda é da mesma ordem que a distância interatômica $\left(\lambda \approx 1 \AA=10^{-10} \mathrm{~m}\right)$, permitindo que ocorram os fenômenos de espalhamento e difração da radiação incidente.

Uma vez que os raios-X formam uma radiação eletromagnética, um raio- $X$ tem um vetor campo elétrico com variação senoidal em relação ao tempo na direção perpendicular à direção de propagação. Esse campo exercerá uma força em uma partícula isolada e carregada, tal como um elétron. De acordo com a teoria clássica, os elétrons, 
agora oscilantes devido ao campo elétrico da radiação incidente, tornam-se uma nova fonte espalhadora em todas as direções. A cristalografia se restringe ao espalhamento elástico, isso é, o comprimento de onda da radiação incidente tem a mesma magnitude do comprimento de onda da radiação espalhada. A amostra na qual a radiação incidente é espalhada, denominada espaço direto. Se a amostra é um cristal, o espaço direto será constituído pela rede cristalina e os eixos cristalográficos a, b e c. O padrão de difração existe em um espaço recíproco ao espaço direto. Dessa maneira, os feixes de Bragg difratados podem ser descritos como pontos do espaço recíproco, sendo constituídos pelos eixos $a^{*}, b^{*}$ e c*. Se a localização dos átomos no espaço direto é definida pelo vetor $\mathrm{r}=\mathrm{xa}+\mathrm{yb}+\mathrm{zc}$, no espaço recíproco, tal localização é dada pelo vetor espalhamento $\mathrm{S}=\mathrm{ha}^{*}+\mathrm{kb}^{*}+\mathrm{lc}^{*}$, onde $\mathrm{h}, \mathrm{k}$ e 1 são os índices de Miller.

O processo de espalhamento, a partir de dois centros, é mostrado na Figura 6 como uma onda plana de um raio$\mathrm{X}$ incidente, sendo espalhado por $\mathrm{a}_{1}$ e $\mathrm{a}_{2}$ e resultando em uma onda plana espalhada. Para isso, assumimos que os espalhadores são comparáveis em tamanho com o comprimento de onda do raio-X, a onda espalhada tem o mesmo comprimento de onda da onda incidente e que os feixes são espalhados uma única vez

Na representação, $s_{0}$ e $s$ são vetores normais às frentes

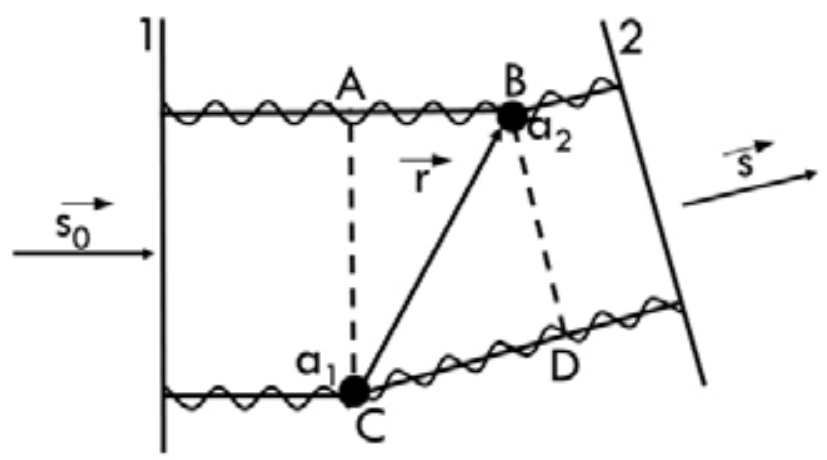

Figura 6. Espalhamentos combinados de dois centros espalhados a1 e a2, situados em C e B; s0 e s são os vetores unitários dos raios incidentes e espalhados, respetivamente e r é a distância entre os espalhadores. de ondas incidente (1) e espalhada (2), com módulos de $\left|s_{0}\right|$ $=|s|=1 / \lambda$, em que $\lambda$ é o comprimento de onda do raio-X. As ondas espalhadas por $a_{1}$ e $a_{2}$ são ditas em fase quando as cristas e vales de onda estão alinhados, resultando em uma amplitude máxima. Para isso, a diferença entre os caminhos dos dois feixes é a diferença das projeções escalares de $\mathrm{r}$ em $s^{0}$ e $s$ e deve ser igual a um número inteiro de comprimento de onda

Em que $S=\left(\mathrm{s}-\mathrm{s}_{0}\right) / \lambda$ é chamado de vetor espalhamento.

$$
\frac{\vec{r} \cdot \vec{s}-\vec{r} \cdot \overrightarrow{s_{0}}}{\lambda}=\vec{r} \cdot \vec{s}
$$

Se é a diferença de caminho para o espalhamento de $\mathrm{a}_{1}$ e $\mathrm{a}_{2}$, então a diferença de fase correspondente é

\section{A LEI DE BRAGG}

$$
\phi=2 \pi \vec{S} \cdot \vec{r}
$$

Em 1912, o fenômeno da difração foi abordado por W. L. Bragg a partir de planos de um retículo cristalino, como pode ser visto na Figura 7. Para isso, consideremos um feixe de raios $\mathrm{X}$ incidente em um par de planos paralelos (Plano 1 e Plano 2). Os raios incidentes formam um ângulo $\theta$ com os planos difratores. Como partículas carregadas, os elétrons oscilarão devido à radiação incidente e espalharão em todas as direções. Para especificamente a direção representada abaixo, onde os raios paralelos secundários emergem em um ângulo $\theta$ como se fosse refletido pelo plano, um feixe espalhado terá sua amplitude máxima, isso é, as ondas estarão em fase 


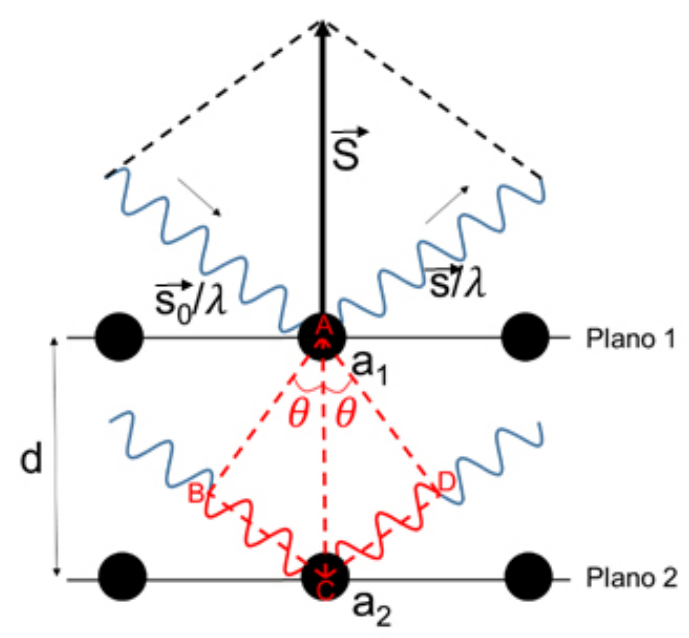

Figura 7: Representação esquemática (fora de escala) da lei de Bragg. S é o vetor espalhamento do espalhador a1. A diferença de caminho ótico entre os planos 1 e 2 é $\mathrm{BC}+\mathrm{CD}$ e está representada na cor vermelha. Os raios-X incidentes formam um ângulo $\theta$ com o plano difrator. Verifica-se a condição de difração quando a diferença de caminho ótico é igual a um múltiplo inteiro do comprimento de onda.

Completando os triângulos $\mathrm{ABC}$ e ACD da Figura 7, torna-se claro que a diferença de caminho ótico entre os planos $B C+C D=2 B C=2 \operatorname{sen} \theta$ é . Para que os feixes continuem em fase, a diferença de caminho ótico deverá ser igual a um múltiplo inteiro do comprimento de onda $\lambda$, dado por

$$
2 d \operatorname{sen} \theta=n \lambda
$$

Um cristal é composto não apenas por um plano de difração, mas por uma família de planos obtidos pela divisão dos eixos a, b e c. Nesse sentido, os índices de Miller hkl relacionam o espaço direto ao espaço recíproco, dividindo os eixos abc em porções iguais, distantes, e onde cada espaçamento representa um nível do plano difrator. Em outras palavras, como a diferença de caminho ótico deve ser igual a um inteiro, o primeiro plano está localizado em ao passo que o plano seguinte mais próximo que satisfaça tal condição deverá cortar o eixo a no segundo espaçamento e terá índice h igual a 2, como representado na Figura 8

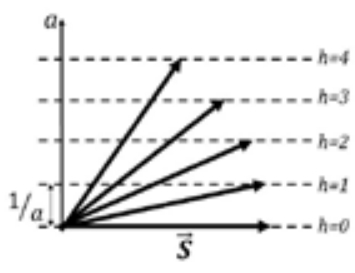

(a)

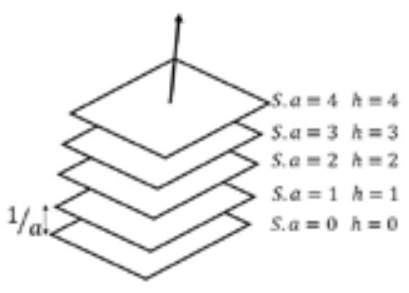

(b)
Figura 8. Representação bidimensional das restrições de Laue para a direção a. As linhas tracejadas indicam a família de planos do espaço recíproco distantes $1 / a$, com níveis $h_{0}, h_{1}, h_{2}, h_{3} e_{4}$. As direções do vetor $S$ satisfazem as condições de Laue (a). (b) representação tridimensional das condições de Laue para a direção a.

As informações supracitadas e representadas na Figura 8 são consequência da explicação para o padrão de difração apresentado por Max von Laue, na qual ele indica que, para que um cristal apresente o máximo de difração, é necessário que sejam satisfeitas três equações simultaneamente

$$
\begin{aligned}
& \vec{s} \cdot \vec{a}=h \\
& \vec{s} \cdot \vec{b}=k \\
& \vec{s} \cdot \vec{c}=l
\end{aligned}
$$

Considerando tanto o espaço direto quanto a reciprocidade desse, a distância interplanar da Figura 7 é representada por $d_{h k l}$ e a Equação 6 torna-se

$$
2 d_{h k l} \operatorname{sen} \theta=n \lambda
$$

em que $n$ é um inteiro. A Equação 8 é conhecida como a lei de Bragg ${ }^{11}$. 


\section{Densidade Eletrônica}

Cada feixe espalhado tem comprimento de onda $\lambda$ e é representado em função de sua amplitude e fase. A amplitude é diretamente proporcional à densidade eletrônica, $\rho(\mathrm{r})$, e a fase é dada em função da direção do vetor espalhamento $\mathrm{S}$ (referente ao espaço recíproco) e do vetor posição $r$ (referente ao espaço direto) do centro espalhador. A função espalhamento $\mathrm{F}(\rightarrow)$ depende do vetor espalhamento e da densidade eletrônica. Uma vez que $\rho(\mathrm{r})$ diz respeito a um único átomo, a função espalhamento fornece o fator de espalhamento atômico $f 12$. O espalhamento da radiação decorrente de um átomo $\mathrm{a}_{\mathrm{j}}$ distante $\mathrm{r}$ da origem da cela unitária é dado por

$$
\mathrm{f}_{\mathrm{j}}=f_{j} \cdot e^{2 \pi i \vec{r}_{j} \cdot \vec{s}}
$$

sendo $\mathrm{f}_{1}=f_{1}$ = fator de espalhamento atômico de aj, quando o centro espalhador está na origem. Representando cada onda espalhada por um vetor, o fator de estrutura $\mathrm{F}$ pode ser obtido pela resultante vetorial das ondas espalhadas individualmente, sendo constituído por um módulo, fase e direção próprios, como na Figura 9

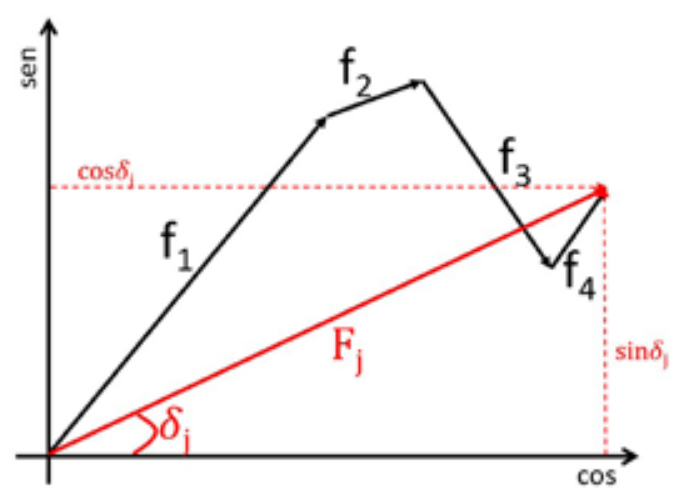

Figura 9. Representação do fator de estrutura resultante da soma das ondas espalhadas $f_{1}, f_{2}, f_{3}$ e $f_{4}$. $O$ eixo das ordenadas representa o seno, enquanto que o eixo das abscissas representa o coseno. em que $f_{1}, f_{2}, f_{3}$ e $f_{4}$ são as ondas espalhadas individualmente e $F_{j}$ é a resultante vetorial das ondas espalhadas, o fator de estrutura. Como visto na imagem acima, o fator de estrutura total de uma cela unitária com $\mathrm{N}$ átomos está decomposto em $\mathrm{N}$ ondas, cada uma com diferentes amplitudes e frequências. À tal decomposição dá-se o nome de Análise de Fourier ${ }^{6}$. O fator de estrutura está constituído por uma componente senoidal e uma componente cosenoidal, sendo seu módulo dado por

$$
|\vec{F}|=\left(x^{2}+y^{2}\right)^{1 / 2}=\left[\left(\sum_{j} f_{j} \cos \hat{\delta}_{j}\right)^{2}+\left(\sum_{j} f_{j} \operatorname{sen} \hat{\delta}_{j}\right)^{2}\right]^{1 / 2}
$$

Considerando que os índices $h k l$ indicam o local de corte dos eixos $a b c$ e que a diferença de caminho ótico diz respeito a sucessivas reflexões de planos sucessivos de uma mesma família (com intervalo de $2 \pi$ ), a diferença de fase em radianos entre a origem e um ponto localizado em $x$, $y, z$ é

$$
\delta=2 \pi(h x+k y+l z)=2 \pi(\vec{r} \cdot \vec{s})
$$

Substituindo a Equação 11 na Equação 10

$|\vec{F}|=\left\{\sum_{j}\left[f f_{j} \cos 2 \pi\left(h x_{j}+k y_{j}+l z_{j}\right)\right]^{z}+\sum_{j}\left[f_{j} \operatorname{sen} 2 \pi\left(h x_{j}+k y_{j}+l z_{j}\right)\right]^{2}\right\}^{2 / 2}$

Considerando, agora, a representação exponencial e complexa do fator de espalhamento de $\mathrm{N}$ átomos da cela unitária, o mesmo pode ser representado por

$F(\vec{s})=\sum_{j=1}^{N} f_{j} \cdot e^{2 \pi i} \vec{r}_{j} \cdot \vec{s}=\sum_{j=1}^{N} f_{j} \cdot e^{2 \pi i\left(h x_{j}+k y_{j}+l z_{j}\right)}$

Finalmente, uma forma generalizada do fator de estrutura é dada em função da densidade eletrônica do cristal. Para isso, aborda-se o espalhamento de todos os elementos infinitesimais da densidade eletrônica de uma cela unitária 


$$
F_{n k t}=\int_{v} \rho\left(x, y_{i} z\right) \cdot e^{2 \pi i\left(k x_{j}+k y_{j}+L z_{j}\right)} d v=\int_{v} \rho(\vec{r}) \cdot e^{2 m(\not 2 \vec{s})} d v
$$

Tão importante quanto o cálculo do fator de estrutura para uma dada distribuição eletrônica é o cálculo da distribuição eletrônica a partir de um dado conjunto de fatores de estrutura, ou seja, o inverso da análise de Fourier. Tal procedimento é feito através da série de Fourier (série de componentes cosenoidais e senoidais ajustados por uma constante $\mathrm{CH}$ que integram uma função periódica desconhecida). A constante, por sua vez, relaciona-se ao fator de estrutura de modo a obter de uma expressão da densidade eletrônica em função do fator de espalhamento, como ilustrado na Equação 15

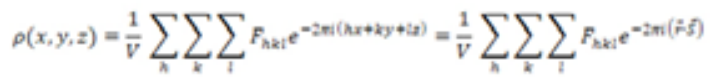

A expressão da densidade eletrônica mostrada na Equação 15 mostra similaridade com a expressão do fator de estrutura da Equação 14. Isso se dá pelo fato de que a primeira é uma expressão para a densidade eletrônica no espaço direto em termos de fator de estrutura no espaço recíproco, enquanto que a segunda representa o fator de estrutura em termos da densidade eletrônica. Em outras palavras, o fator de estrutura é a transformada de Fourier da densidade eletrônica e a densidade eletrônica é a transformada de Fourier inversa do fator de estrutura, evidenciado pelo sinal negativo da exponencial ${ }^{5}$. Uma expressão alternativa para a expressão de densidade eletrônica da Equação ${ }^{15}$ pode ser obtida partindo da forma polar do fator de estrutura

$$
F_{h k l}=\left|F_{h k l}\right| e^{i \alpha_{h k l}}=\left|F_{h k l}\right| e^{2 \pi i \alpha_{h k l}^{\prime}}
$$

e substituindo a Equação 16 na Equação 15

$$
\rho(x, y, z)=\frac{1}{V} \sum_{h} \sum_{k} \sum_{l} F_{h k l} e^{-2 \pi i\left(h x+k y+l z-\alpha_{h k l}^{\prime}\right)}
$$

em que $\alpha$ 'hkl é o ângulo de fase em radianos. A Equação 17 é vantajosa, uma vez que o ângulo de fase $2 \pi \alpha^{\prime}{ }_{\text {hkl }}$ para cada coeficiente aparece explicitamente ${ }^{4}$.

A exposição do cristal aos raios- $\mathrm{X}$ resulta em um conjunto de amplitude das ondas espalhadas $\left|F_{h k l}\right|$, mas o conjunto de fases correspondentes $\alpha$ 'hkl não podem ser definidos diretamente pelo experimento. $\mathrm{O}$ conhecimento das fases dos fatores de estrutura é de vital importância para calcular a síntese de Fourier da densidade eletrônica por meio da Equação 17. Assim sendo, a estrutura cristalina não pode ser determinada por experimentos diretos, uma vez que a formação da imagem correspondente não ocorre e, também não pode ser obtida diretamente por cálculos devido ao desconhecimento das fases relativas às ondas da radiação. A estrutura cristalina é determinada indiretamente partindo-se do padrão de difração, procedimento no qual as fases são perdidas. Logo, fica evidenciado o problema da fase, visto que a determinação da densidade eletrônica depende do conhecimento das fases perdidas durante o processo de medida ${ }^{13}$.

\section{Conclusão}

Nesse , foram detalhados os três pilares da teoria básica cristalográfica (simetria, difração de raios-x e densidade eletrônica). É claro que uma elucidação estrutural envolve outros aspectos não abordados aqui, mas os conteúdos explanados são de vital importância na compreensão da metodologia cristalográfica. Dessa maneira, espera-se que tais conteúdos auxiliem na correlação teoria versus prática envolvida em análises dessa natureza.

\section{Agradecimentos}

Os autores agradecem a agência de fomento à pesquisa de Coordenação de Aperfeiçoamento de Pessoal de Nível Superior (CAPES) pelo apoio financeiro para a realização desse trabalho. 


\section{Artigo Convidado 3}

\section{Referências}

1. N. G. Szwacki and T. Szwacka, Basic Elements of Crystallography, Pan Stanford Publishing Pte. Ltd., Singapore, 2010.

2. B. D. Cullity, Am. J. Phys., 1957, 25, 394.

3. C. Hammond, The Basics of Crystallography and Diffraction, Oxford University Press, Leeds, 4th edn., 2015.

4. L. H. Jensen and G. H. Stout, X-Ray Structure Determination: A Practical Guide, Second Edition, Seattle, Washington, 2nd edn., 1990, vol. 67.

5. C. Giacovazzo, H. L. Monaco, G. Artioli, D. Viterbo, M. Milanesio, G. Gilli, P. Gilli, G. Zanotti, G. Ferraris and M. Catti, Fundamentals of Crystallography, Oxford University Press, Oxford, 3rd edn., 2011.

6. J. P. . Glusker, M. Lewis and M. Rossi, Crystal Structure Analysis for Chemists and Biologists, New York, 1st edn., 1994, vol. 1.

7. S. K. Chatterjee, Crystallography and the World of Symmetry, Springer Berlin Heidelberg, Berlin, Heidelberg, 1st edn., 2008, vol. 113.

8. P. Paufler, Acta Crystallogr. Sect. A, 2004, 60, 641-642.

9. P. Atkins and L. Jones, Chemical Principles - The Quest for Insight, W. H. Freeman, New York, Fifth Edit., 2010.

10. D. Halliday, R. Resnick and J. Walker, Fundamentals of Physics Extended, Wiley, Hoboken, NJ, USA, 10th edn., 2013.

11. P. H. Napolitano, A. Camargo and Y. Mascarenhas, 2007, 35-45.

12. A. a. Bunaciu, E. G. Udriştioiu and H. Y. Aboul-Enein, Crit. Rev. Anal. Chem., 2015, 45, 289-299.

13. G. Taylor, Acta Crystallogr. Sect. D Biol. Crystallogr., 2003, 59, $1881-1890$

\section{Jean Marcos F. Custodio ${ }^{a, b^{*}}$, Luiz F. N. Naves ${ }^{b}$, Wesley F. $\mathrm{Vaz}^{\mathrm{b}, \mathrm{c}}$}

${ }^{a}$ Instituto de Química, Universidade Federal de Goiás, 74690-900, Goiânia - GO, Brasil

${ }^{\mathrm{b}}$ Ciências Exatas e Tecnológicas, Universidade Estadual de Goiás, 87020900, Anápolis - GO, Brasil

' Instituto Federal de Educação, Ciência e Tecnologia de Mato Grosso, 78455-000 Lucas do Rio Verde-MT, Brasil

*e-mail: jeanmfcustodio@gmail.com 